\title{
The (In)Effectiveness of Animation in Instruction
}

\author{
Julie B. Morrison and Barbara Tversky \\ Department of Psychology \\ Jordan Hall, Building 420 \\ Stanford, California 94305-2130 \\ $+1650725-2400$ \\ \{julie,bt\}@psych.stanford.edu
}

\begin{abstract}
Animated graphics have been increasingly adopted to teach complex systems, encouraged by the preconception that realism is effective. Nevertheless, the evidence has been discouraging as to their effectiveness. By the Conceptual Congruence Hypothesis, graphics should be effective in conveying concepts that are literally or metaphorically spatial. By extension, animated graphics should be effective in conveying change in time. This hypothesis was investigated by comparing three interfaces that presented text, text plus static graphics, or text plus animated graphics. Evidence was obtained for the static version of the Conceptual Congruence Hypothesis. Graphics were more effective than text in some cases, especially for participants with low spatial ability, but animation did not further increase effectiveness.
\end{abstract}

\section{Keywords}

Animation, graphics, multimedia, interfaces, instruction

\section{INTRODUCTION}

Each type of presentation format, text, graphic, and animation, has its own strengths in conveying information. Language is idea for conveying causal and conditional relations as these are difficult to depict. Additionally, language can convey concrete concepts that are easy to visualize without external aids. Graphics, however, may convey complex conceptual information by showing system components in relation to one another. All components of a conceptual system can be displayed together, providing both an overview of the entire structure and all possible relations. Furthermore, space can be used metaphorically in graphics, for example, to represent the causal structure of a system.

Animations are claimed to have an advantage over text and static graphics in showing change in time. This can be literal, in that animation can use motion to convey motion. It can be metaphoric in using change in graphics to show change in processes. Thus, animation may be the most congruent way to convey conceptual information about motion or change. The hypothesis that certain media are naturally more effective for conveying certain types of information, will be referred to as the Conceptual Congruence Hypothesis.

The static version of the Conceptual Congruence Hypothesis has received support from research examining mapping spatial and metaphorically spatial information onto static space [e. g., 2, 4]. The greater the congruence between the presentation medium (i.e., a graphic) and the information, the greater the facilitation. The Conceptual Congruence Hypothesis can be extended to animation in the following way: Because animations use motion to show change over time, presenting information about change over time with animation is most congruent and should facilitate learning [e.g., 1]. The present research evaluates both static and dynamic versions of the Conceptual Congruence Hypothesis.

\section{METHOD}

Participants

One hundred and forty-four Stanford University students participated in this series of experiments with participants divided equally among three presentation media.

\section{Learning Interface}

The three experiments compared the learning of seven rules of movement presented as informationally equivalent Text, Text Plus Static Graphics, or Text Plus Animated Graphics. In the first experiment, the text included the causal rationale for the rules of movement with movement explained as social rules or as navigation rules. In the second experiment, the rationale was omitted and only the rules were described. The third experiment used the same materials as the second but limited participants' study time. A sample of the interface is available at http://wwwpsych.stanford.edu/ julie/interface.html.

\section{Procedure}

Participants completed a test of spatial ability, the Vandenberg (revised version, 1979) mental rotation test, and then began the learning phase of the experiment. In the first two experiments, participants read the rules four times, each time for as long as they desired. In the third experiment, participants saw the rules only once and were pressured to study quickly. Following the learning phase of the experiment, participants completed three timed performance tests requiring applications of the rules. The scores on these tests have been combined into a composite Problem-Solving score which could range from 0-100.

\section{RESULTS AND DISCUSSION}

Analyses were conducted after dividing participants into groups according to spatial ability. Participants who scored

(C) Copyright on this material is held by the Author(s). 
below the mean on the spatial ability test were placed in the low spatial ability group, while those participants who scored above the mean were placed in the high spatial ability group. As the grouping was based on Stanford undergraduates, it may not reflect ability in the general population.

The first experiment supported the static version of the Conceptual Congruence Hypothesis for low spatial ability participants (Figure 1). However, text with animated graphics did not enhance learning over and above text with static graphics.

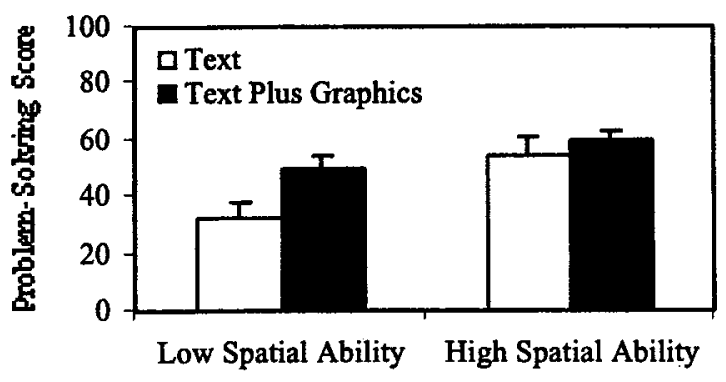

Figure 1. The relation between spatial ability and presentation medium in Experiment 1. The Experiment 2 pattern is identical. The Text Plus Graphics condition combines both static and animated graphics conditions as these did not differ.

In the second experiment, the importance of the graphics for learning was increased by eliminating causal explanations for the rules. Participants had to develop their understanding of this system and its dynamics solely from descriptions of the movement allowed in the system. However, this manipulation did not change the results. As with the first experiment, graphics were superior to text only for low spatial ability participants and animated graphics were not superior to static ones.

Closer analysis of the first two experiments suggests that congruence effects may be diminished when there is sufficient time to learn the text. The third experiment investigated this hypothesis, that congruence is more important when learning time is limited, by presenting the same materials as in the second experiment but limiting study times. For all participants, the more congruent medium facilitated performance, that is, text plus graphics was superior to text alone. However, as before, animation did not enhance performance further. With restricted study time, low spatial ability participants in both the Text and the Text Plus Graphics conditions performed more poorly than their high spatial ability counterparts. In the first two experiments with unlimited study time, low spatial ability participants with graphics performed as well as high spatial ability participants (Figure 2).

These data support the static graphic version of the Conceptual Congruence Hypothesis. However, presenting information dynamically was never more successful than presenting the same information spatially with only implied dynamics, even though the information to be learned was the dynamics of the system.

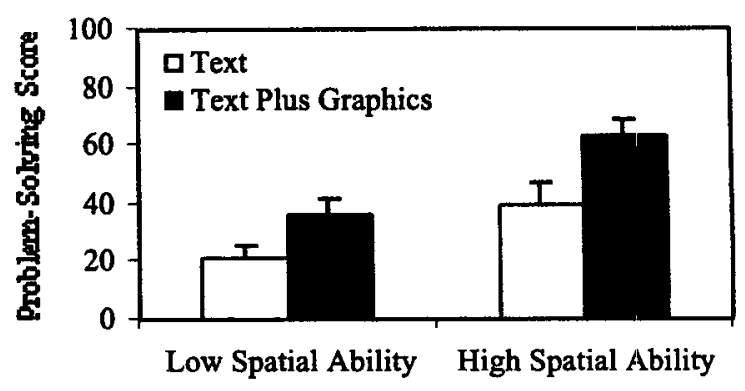

Figure 2. The relation between spatial ability and presentation medium in Experiment 3.

\section{CONCLUSIONS}

This research has provided evidence for the role of congruence of information in the facilitation of learning; namely, that graphics convey spatial information better than text alone because they represent space with space. Although animated graphics use motion to show change over time, presenting information about change over time with animated graphics did not enhance learning beyond presenting static graphics. This failure to show facilitation of animated over static graphics is consistent with the numerous other failures, and not a single clear success, reported by researchers in HCI, psychology, and education [e.g., 3]. Despite their conceptual congruence, arimations may not facilitate learning because they may be hard to perceive and because people conceive of dynamic systems as sequences of steps [e.g., 3]. Of course, it is possible that animations may be effective in other situations, especially for maintaining users' attention and comprehension on-line, as in zooming.

\section{ACKNOWLEDGMENTS}

This work was supported by a Varenius grant from the National Center for Geographic Information and Analysis and by an Office of Naval Research grant No. NOOO14-001-0649.

\section{REFERENCES}

1. Kieras, D. E. (1992). Diagrammatic displays for engineered systems: Effects on human performance in interacting with malfunctioning systems. International Journal of Man-Machine Studies, 36, 861-895.

2. Mayer, R. E. (1989). Models for understanding. Review of Educational Research, 59, 43-64.

3. Morrison, J. B., Tversky, B., \& Betrancourt, M. (2000). Animation: Does it facilitate learning? Proceedings of Workshop on Smart Graphics. Menlo Park: AAAI Press.

4. Tversky, B. (1995). Cognitive origins of graphic conventions. In F. T. Marchese (Ed.). Understanding images (pp. 29-53). New York: Springer-Verlag. 